\title{
Orbital decompression for inverted papilloma - Case report
}

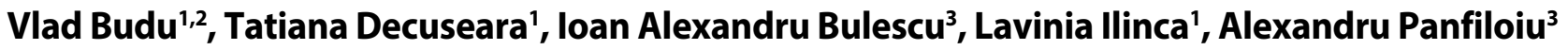 \\ 1"Prof. Dr. Dorin Hociota" Institute of Phono-Audiology and Functional ENT Surgery, Bucharest, Romania \\ 2“Carol Davila” University of Medicine and Pharmacy, Bucharest, Romania \\ ${ }^{3}$ Medicover Hospital, Bucharest, Romania
}

\section{ABSTRACT}

Inverted papilloma is a rare benign sinonasal tumor with a still unclear etiology. In the last decades, HPV implication in inverted papilloma etiopathogeny has been studied and demonstrated in some scientific research papers, but with no causality proven. Imagistic investigations and the Krouse classification establish the surgical approach. Endoscopic surgery represents the golden standard treatment for most cases of inverted papilloma, while external or combined approaches present limited indications.

The authors present a case and therapeutic plan of an inverted papilloma located in the ethmoid sinus, with origin in the lamina papyracea and high-risk HPV implication.

KEYWORDS: inverted papilloma, HPV, FESS.

\section{INTRODUCTION}

Inverted papilloma is a benign sinonasal tumor, most commonly unilateral, best known for its potential of recurrence and tendency to malignant transformation. It is an uncommon tumor, with a percentage of $0.5-4$ of all primary nasal tumors ${ }^{1}$. Histologically, inverted papilloma (IP) is an epithelial growth into the underlying connective tissue stroma. Even though it is a benign tumor, inverted papilloma often has an invasive evolution, associated with destruction of bony walls and extension into adjacent structures. It is most frequently diagnosed in adulthood, in the fifth decade of life, with a male to female ratio of 2-5:12.

In 1854, Ward first observed inverted papilloma located in the sinonasal cavity, but its histological nature was described in 1930s by Reingertz and, in 1971, Hymans classified sinonasal papilloma into inverted, fungiform and cylindrical ${ }^{1}$. Currently, inverted papilloma is considered a sinonasal Schneiderian papilloma and, since 2005, it is classified by the World Health Organization into inverted, oncocytic and exophytic papilloma, with inverted papilloma presenting the highest occurrence rates of $62 \%$, followed by exophytic papilloma with $32 \%$ and oncocytic papilloma with $6 \%{ }^{2}$.

Etiology of inverted papilloma has not yet been fully understood. Since late 1980s, studies have shown a close relationship with viral infections, in particular with HPV (Human Papilloma Virus). Although it is the most studied, HPV infec- tion in inverted papilloma presents variable detection rates between different studies. Viral implication has been considered because of IP's tendency to recurrence and potential for malignant transformation ${ }^{2}$. The most frequent serotypes of HPV are 6 and 11, while high-risk types 16 and 18 are found in IP with high-grade dysplasia or carcinoma ${ }^{2}$.

Symptomatology is nonspecific, with nasal obstruction, rhinorrhea, headache, epistaxis and hyposmia or anosmia and can be uni- or bilateral, depending on the tumor location. Diagnostic of IP must include a clinical examination, imagistic investigation and histopathological examination. The endoscopic examination typically shows a unilateral irregular tumor mass, with bleeding tendency, frequently accompanied by inflammatory polyps. Any patient with unilateral lesions should be suspected of inverted papilloma. The imagistic investigation consists in computed tomography and, if needed, an MRI. Computed tomography (CT) is the gold standard investigation, as it illustrates tumor extension, the relationship with surrounding structures and tumor origin. Tumor origin can be observed on CT images as focal hyperostosis and its identification can influence the therapeutic approach. MRI complements CT scans, as the later cannot differentiate the tumor from inflammatory tissue, though it is rarely used as a basic investigation.

Treatment for inverted papilloma is exclusively surgical and consists in complete excision of the tumor. Surgical indication must take into account imagistic characteristics 

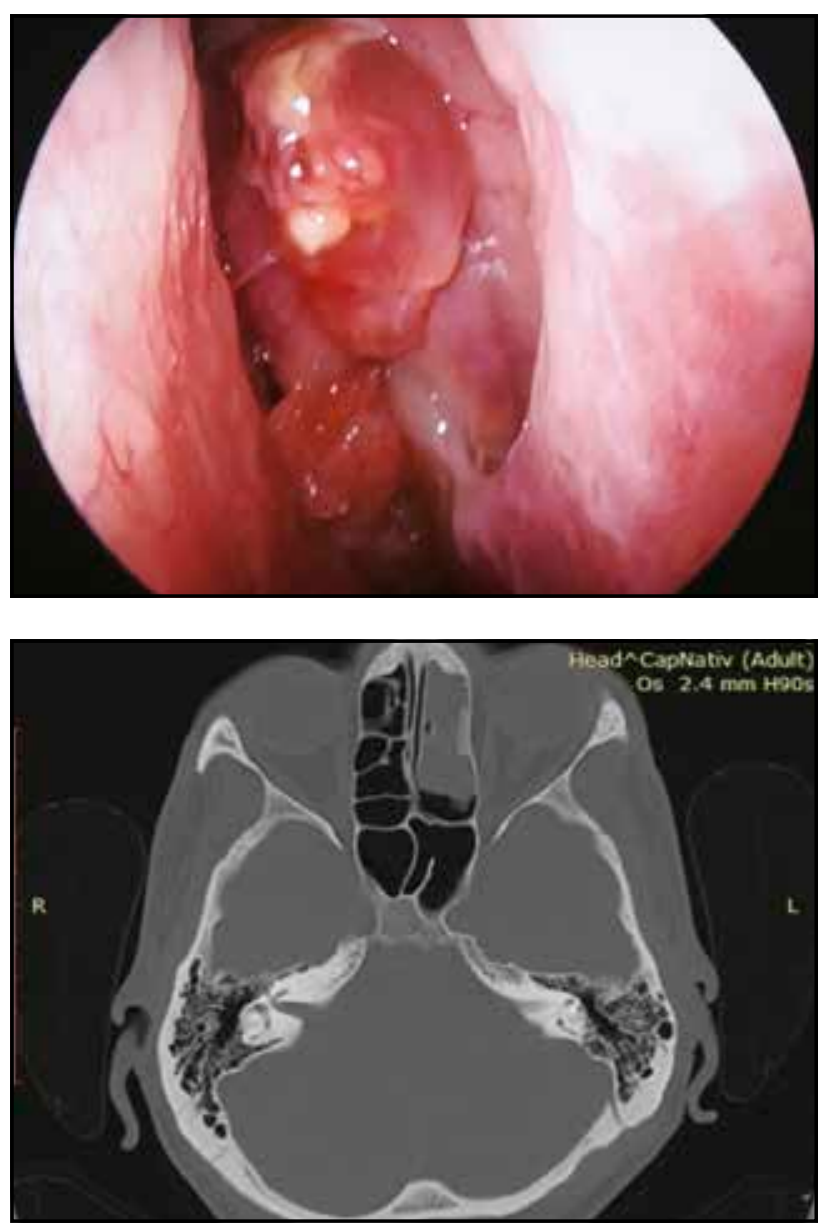

Figure 1 Endoscopic and CT aspect.

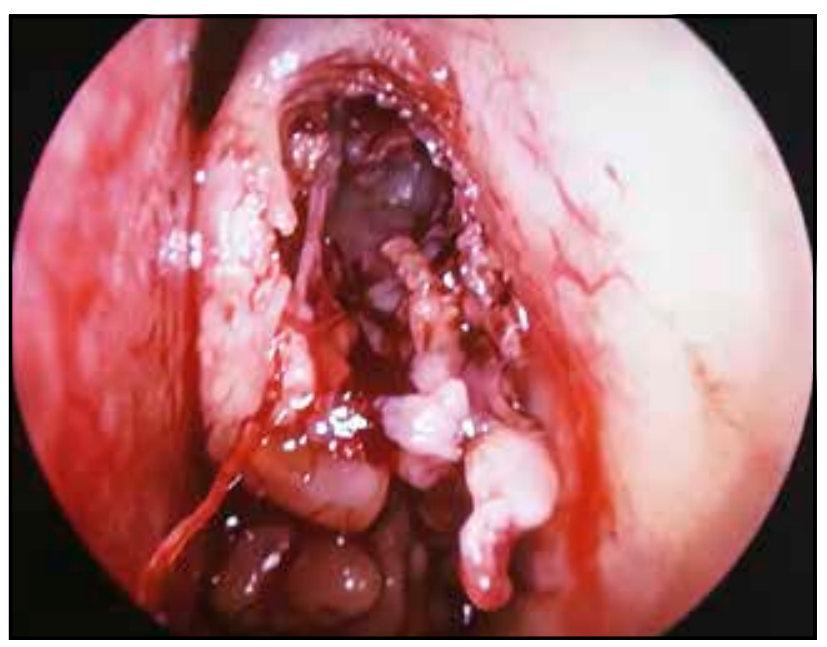

Figure 2 Intraoperative aspect - anterior ethmoidectomy with a complete removal of the tumor.

and the Krouse classification. Its main goals are tumor removal and ensuring normal sinus ventilation. For decades, surgery was performed by an open approach, which, depending on the tumor location, implied a paralateronasal approach or midfacial-degloving with medial maxillectomy. Endoscopic surgery is, nowadays, the gold standard treatment for inverted papilloma. In addition to all advantages of endoscopy, such as minimally invasive technique, superior aesthetic results, it ensures tumoral excision control by providing a better view of the mucosa and has proven to present low recurrence rates ${ }^{3}$. Because of IP's aggressive nature, it can present orbital and cranial complications and has a 5 to $15 \%$ chance of malignant transformation ${ }^{3}$. Besides viral implication, recurrence can be secondary to residual tumor and appears in $12 \%$ of the cases ${ }^{4}$.

\section{CASE REPORT}

The authors present the case of a 45 -year-old male patient, who was admitted in our clinic for left chronic nasal obstruction and frontal headache, with onset from about 7 months earlier, which progressively aggravated.

The diagnostic protocol in our clinic includes an endoscopic exam, computed tomography and histopathological examination. Endoscopy revealed an irregular, papillomatous tumor mass, which completely blocked the left middle meatus, and sero-mucous secretions. CT imaging showed the left ethmoid sinus completely opacified and a zone of hyperostosis situated on the left lateral nasal wall (lamina papyracea) (Figure 1).

Relying on CT images and endoscopic exam, the suspicion of an inverted papilloma emerged and it was graded according to the Krouse classification into a stage T2 tumor. We decided for an endoscopic surgical approach with complete resection of the tumor, followed by removal of the adjacent mucosa and started drilling the vicinity bone. An anterior ethmoidectomy was performed and the tumor was completely removed (Figure 2).

We successfully identified the origin site of the tumor on the lamina papyracea and decided to perform a complete resection of the adjacent bone. We considered that drilling this bony part would be unsafe, associated with a high risk of recurrence, so we performed an orbital decompression in which the lamina papyracea of the anterior ethmoid was resected (Figure 3).

After we practiced orbital decompression, we identified a left anterior ethmoid artery branch with orbital origin. This vascular structure was discovered after the bony origin of the papilloma had been resected, and lay on the anterior face of the basal lamella, superiorly, passing through the periorbita. The anterior ethmoidal artery developed small branches for the tumor, which we decided to cauterize and ended on the lateral wall of the left middle turbinate (Figure 4).

The result consisted in a complete macroscopic excision of the tumor with resection of the underlying mucosa and lamina papyracea. Because of the ethmoidal location of the tumor, there was no use in performing an endoscopic middle maxillectomy, even if we checked the maxillary sinus. At the end of the surgery, the left periorbita was completely tumorfree and could be seen lying in the osteomeatal unit with no 

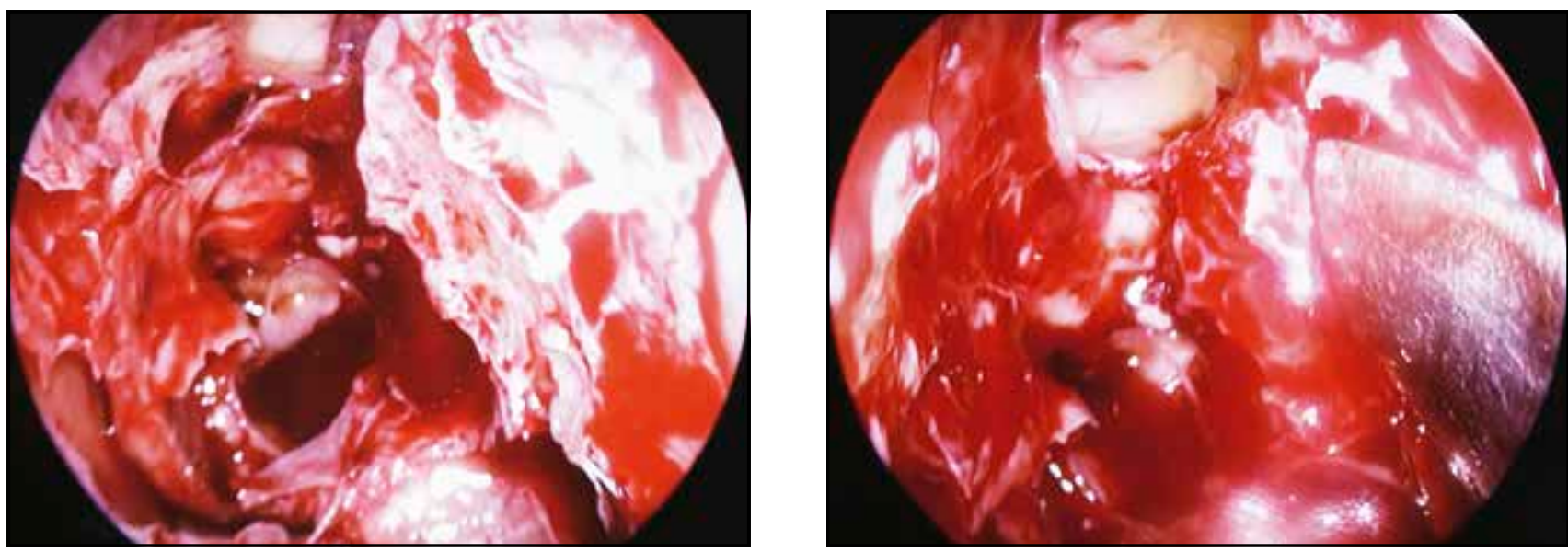

Figure 3 Orbital decompression - intraoperatory view.
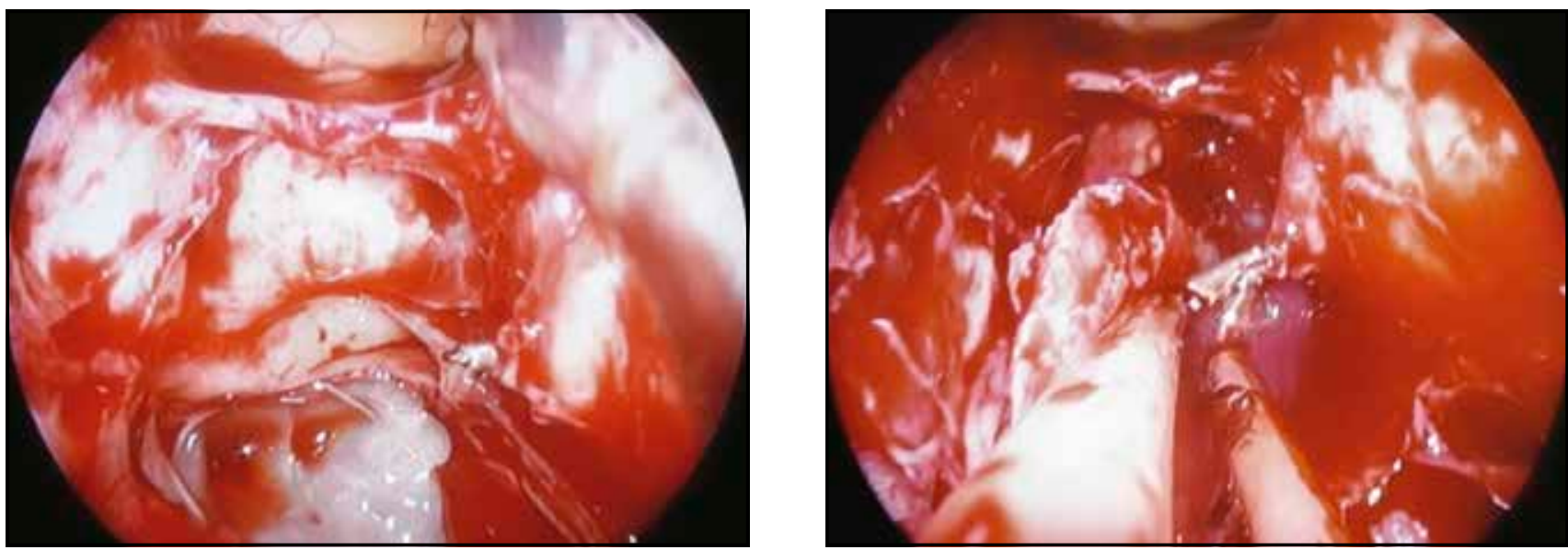

Figure 4 The left anterior ethmoid artery.

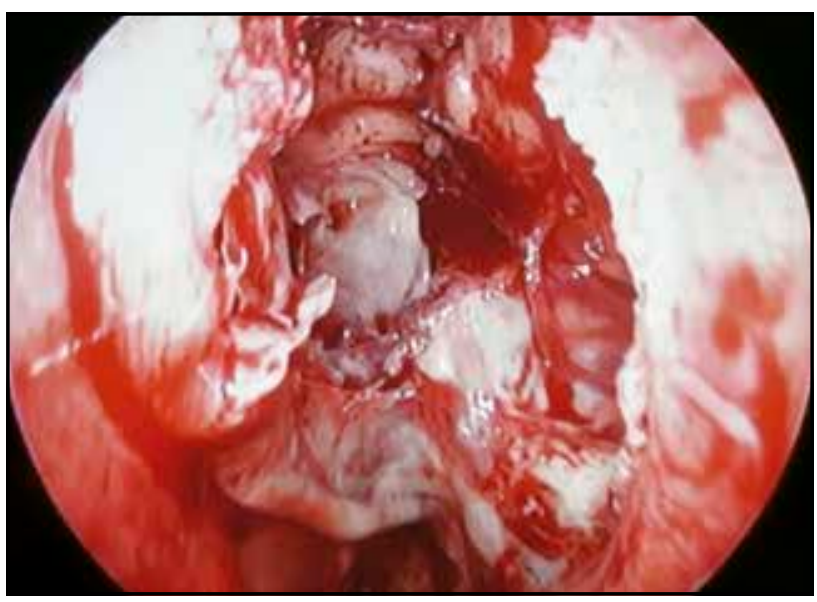

Figure 5 Final intraoperative view: the middle meatus, the ethmoid artery and the periorbita.

protrusion of the left orbital fat (Figure 5). The surgery did not require anterior nasal packing and we used only a resorbable sponge for filling the middle meatus.
The tumor was sent for histopathological examination and the result confirmed the diagnostic of inverted papilloma. Immunohistochemistry examination revealed viral implication with high-risk type $16 \mathrm{HPV}$. After hospital discharge, the patient followed a treatment with Isoprinosine for 6 months. Follow-up was thorough; in the first 6 months, it was performed on a monthly basis and at three months until one year after the surgery. In the future, the follow-up plan for the patient consists in biannual ENT examination for the second and third year after surgery and once a year for years 4 and 5 after surgery. A CT examination will be recommended if the patient develops any signs of suspicion for recurrence.

\section{DISCUSSIONS}

Inverted papilloma is a challenging pathology because of its tendency to recurrence, invasive nature and possibility of malignancy. Etiology is still unclear, though there have been mentioned some risk factors, with no causality proven, such as chronic inflammation, smoking and viral infections ${ }^{5}$. Despite the advances made in detection of the 
viral genome in the last decades, viral implication in IP has not been demonstrated and fully accepted yet. Even so, many studies have proven the association of HPV and inverted papilloma, in up to $40 \%$ of the cases ${ }^{2}$. Studies have demonstrated that low-risk types 6 and $11 \mathrm{HPV}$ are more frequently implicated in inverted papilloma, than highrisk types 16 and 18. From all forms of papilloma, HPV is more often detected in exophytic papillomas than $\mathrm{IP}^{6}$.

The diagnostic must include anamnesis (unilateral symptoms, history of recurrent epistaxis), endoscopy, imagistic examination (CT and MRI) and histopathological exam, completed by immunohistochemistry testing. Krouse classification directs the surgical approach ${ }^{7}$. Starting from this classification, stage $\mathrm{T} 1$ and $\mathrm{T} 2$ inverted papilloma can be treated endoscopically, while stage T3 and T4 might require a combined or external approach ${ }^{5}$. Besides the Krouse classification, another important factor in choosing a surgical approach is identifying the point of origin.

Elective treatment for inverted papilloma is represented by complete resection of the tumor. Endoscopic surgery has the superior advantage of a better view of the tumoral limits, normal mucosa and other endoscopic landmarks. Complete resection is essential for therapeutic management, considering the risk of recurrence and malignant transformation. Recurrence of the inverted papilloma can either be early, or late. International literature describes occupational exposures and smoking as risk factors for recurrence and dysplasia, but not for tumor occurrence ${ }^{5}$. Also, according to the Krouse classification, a stage T3 IP presents a 51\% higher risk of recurrence, as it has been shown in a recent study ${ }^{8}$. Malignancy is the main concern for this pathology, as in $8 \%$ of the cases recurrence occurs in a malignant form ${ }^{3}$.

Other therapeutic approaches include an antiviral treatment with Isoprinosine or Interferon, intralesional administration of Cidofovir or anti-angiogenic treatment with Bevacizumab.

In our case, we opted for performing endoscopic surgical resection of the tumor with orbital decompression, considering the extended zone of hyperostosis showed on CT images and located on the lamina papyracea. The intraoperative risk was represented by a branch of the left anterior ethmoidal artery, which we decided to cauterize in order to avoid intraoperative or postoperative haemorrhage. Medical treatment consisted in a 6-month therapy with Isoprinosine, in order to lower the risk of recurrence. Histopathology and immunohistochemistry revealed a high-risk type $16 \mathrm{HPV}$ infection.

This case emphasizes the importance of HPV genotyping in the inverted papilloma diagnostic algorithm ${ }^{9}$. The question remains as to what is the next therapeutic attitude for an inverted papilloma with macroscopically complete resection, but with type $16 \mathrm{HPV}$ determination. When dealing with a high-risk HPV infection, a "wait-and-see" strategy is unsafe, as the potential of malignant transformation is real. This also highlights the need of a serologic marker for inverted papilloma and its malignancy potential. There are several tests in research, such as SCCA (squamous cell carcinoma antigen), Ki-67, BCL-2 and Wnt proteins, but with no current practical usage for this pathology5.

\section{CONCLUSIONS}

Inverted papilloma is a challenging pathology in ENT because of the risks that can occur. It is a benign tumor, with viral etiology, and has an aggressive nature, a tendency to recurrence and potential for malignant degeneration. The gold standard treatment is surgery and is guided by the Krouse classification. Most cases of recurrence are caused by residual tumor. This is why surgical treatment must imply radical excision of the tumor and, when an origin point is identified, usage of a burr in order to eliminate it. HPV implication must be taken into consideration when deciding a therapeutic plan and determining the viral genotype must be included in basic testing. Further research is to be made into the possibility of predicting the malignant potential of any case of inverted papilloma.

Conflict of interest: The authors have no conflict of interest.

Contribution of authors: All authors have equally contributed to this work.

\section{REFERENCES}

1. Khandekar S, Dive A, Mishra R, Upadhyaya N. Sinonasal inverted papilloma: A case report and mini review of histopathological features. J Oral Maxillofac Pathol. 2015;19(3):405

2. Lisan Q, Laccourreye O, Bonfils P. Sinonasal inverted papilloma: From diagnosis to treatment. Eur Ann Otorhinolaryngol Head Neck Dis. 2016;133(5):337-41. DOI: 10.1016/j.anorl.2016.03.006. Epub 2016 Apr 1.

3. Ungari C, Riccardi E, Reale G, Agrillo A, Rinna C, Mitro V, et al. Management and treatment of sinonasal inverted papilloma. Ann Stomatol (Roma). 2016;6(3-4):87-90. DOI: 10.11138/ads/2015.6.3.087. eCollection 2015 Jul-Dec.

4. Busquets JM, Hwang PH. Endoscopic resection of sinonasal inverted papilloma: a metaanalysis. Otolaryngol Head Neck Surg. 2006;134(3):476-82.

5. Gamrot-Wrzoł M, Sowa P, Lisowska G, Scierski W, Misiolek M. Risk factors of recurrence and malignant transformation of sinonasal inverted papilloma. [Internet]. Biomed Res Int. 2017;2017:9195163. Published online 2017 Nov 9. DOI: 10.1155/2017/9195163. Available from: https://www.ncbi.nlm.nih.gov/pmc/articles/PMC5700512/.

6. Lawson W, Schlecht NF, Brandwein-Gensler M. The role of the human papillomavirus in the pathogenesis of Schneiderian inverted papillomas: an analytic overview of the evidence. Head Neck Pathol. 2008;2(2):49-59. DOI: 10.1007/s12105-008-0048-3. Epub 2008 Apr 23.

7. Oikawa K, Furuta Y, Nakamaru Y, Oridate N, Fukuda S. Preoperative staging and surgical approaches for sinonasal inverted papilloma. Ann Otol Rhinol Laryngol. 2007;116(9):674-80.

8. Lisan Q, Moya-Plana A, Bonfils P. Association of Krouse Classification for Sinonasal Inverted Papilloma With Recurrence: A Systematic Review and Meta-analysis. JAMA Otolaryngol Head Neck Surg, 2017;143(11):1104-10. DOI: 10.1001/jamaoto.2017.1686.

9. Budu V, Schnaider A, Bulescu I. Endoscopic approach of sinonasal inverted papilloma - our 15 years experience on 162 cases. Rom J Rhinol. 2015;5(17):31-6. DOI: 10.1515/ rjr-2015-0004. 